\title{
DISCOVERY OF THE FIRST GIANT DOUBLE RADIO RELIC IN A GALAXY CLUSTER FOUND IN THE PLANCK SUNYAEV-ZEL'DOVICH CLUSTER SURVEY: PLCK G287.0+32.9
}

\author{
Joydeep Bagchi ${ }^{1}$, S. K. Sirothia ${ }^{2}$, Norbert Werner ${ }^{3}$, Mahadev B. Pandge ${ }^{4}$, Nimisha G. \\ Kantharia $^{2}$, C.H. Ishwara-Chandra ${ }^{2}$, Gopal-Krishna ${ }^{2}$, Surajit Paul ${ }^{1}$ and Santosh Joshi ${ }^{5}$
}

\begin{abstract}
We report the discovery of large-scale diffuse non-thermal radio emission in PLCK G287.0+32.9, an exceptionally hot $(T \sim 13 \mathrm{keV})$, massive, and luminous galaxy cluster, strongly detected by the Planck satellite in a recent, all-sky blind search for new clusters through Sunyaev-Zel'dovich effect. Giant Metrewave Radio Telescope $150 \mathrm{MHz}$ and Very Large Array 1.4 GHz radio data reveal a pair of giant (>1 Mpc) "arc" shaped peripheral radio-relics (signatures of shock waves) of unprecedented scale (linear separation $\sim 4.4 \mathrm{Mpc}$ at redshift 0.39), located at distances from the cluster center which are about 0.7 and 1.3 of the cluster's virial radius. Another possible giant relic and a radio-halo is detected closer to the cluster center. These relic sources are unique "signposts" of extremely energetic mergers and shocks (both ongoing and past), that are assembling and heating up this very massive galaxy cluster. They are also a probe of the filamentary cosmic-web structure beyond the cluster virial radius. Optical imaging with the IUCAA $2 \mathrm{~m}$ telescope and XMM-Newton X-ray data confirm a very rich galaxy cluster with a morphologically disturbed core region, suggesting a dynamically perturbed merging system.
\end{abstract}

\footnotetext{
${ }^{1}$ The Inter-University Centre for Astronomy and Astrophysics (IUCAA), Pune University Campus, Post Bag 4, Pune 411007, India; joydeep@iucaa.ernet.in

${ }^{2}$ National Centre for Radio Astrophysics, TIFR, Pune University Campus, Post Bag 3, Pune 411007, India

${ }^{3}$ Kavli Institute for Particle Astrophysics and Cosmology, Stanford University, 452 Lomita Mall, Stanford, CA 94305, USA

${ }^{4}$ School of Physical Sciences, Swami Ramanand Teerth Marathwada University, Vishnupuri, Nanded 431606, India

${ }^{5}$ Aryabhatta Research Institute of Observational Sciences (ARIES), Manora Peak, Nainital 263129, India
} 
Subject headings: cosmology: observations - galaxies: clusters: individual(PLCK G287.0+32.9) - shock waves - X-rays: galaxies: clusters

\section{Introduction}

Two main baryonic constituents of the diffuse intracluster medium (ICM) are the diffuse hot $\left(T \sim 10^{7}-10^{8} \mathrm{~K}\right)$, tenuous $\left(n_{0} \sim 10^{-(3-4)} \mathrm{cm}^{-3}\right)$ ICM and a rarely visible nonthermal population of extremely high energy relativistic particles. The presence of $\mathrm{GeV}$ electrons/positrons (of largely unknown origin) is inferred from the diffuse synchrotron radio emission in a fraction of galaxy clusters, which lacks an obvious association with any of the cluster galaxies. These radio sources, which usually possess large size $(\sim 0.1-1 \mathrm{Mpc})$ and steep spectra $\left(\alpha \sim 1-3 ; \mathrm{S}_{\nu} \propto \nu^{-\alpha}\right)$, are called "radio halos" if they permeate the cluster centers and "radio relics" if they are located at the cluster peripheral regions (e.g., the review by Ferrari et al. 2008).

Since radio halos and relics are exclusively found in the clusters clearly showing Xray or optical substructure (Govoni et al. 2004), their origin is related to the merging processes of galaxy clusters, although its details are still obscure. Signatures of giant shock waves arising from cluster mergers have been discovered in recent years (Bagchi et al. 2006; van Weeren et al. 2010), and such collisionless shocks are capable of accelerating the particles to relativistic energies, giving rise to the radio relics via in-situ diffusive shock acceleration of electrons (primary electrons). The radio halos may arise via turbulent energy injection or in-situ acceleration of protons with the secondary production of relativistic

electrons and positrons by inelastic $p-p$ collisions (secondary electrons) (Dennison 1980; Brunetti and Lazarian 2007).

The free thermal electrons of hot ICM inverse Compton scatter the photons of cosmic microwave background (CMBR). This results in a predictable spectral distortion of CMBR toward the cluster which is the well-known thermal Sunyaev-Zel'dovich (SZ) effect (Sunyaev and Zel'dovich 1972; Sunvaev and Zel'dovich 1980). The SZ effect is an exceptional tool for the study of clusters as the surface brightness of SZ signal is independent of redshift and depends only on the integrated thermal pressure of ICM along the line-of-sight. The total SZ signal, being a measure of thermal energy of the cluster, tightly correlates with the cluster mass, making it a powerful, unbiased tool for making volume limited samples and for finding the high redshift and the most massive clusters (Vanderlinde et al. 2010; Williamson et al. 2011). Of particular interest is to investigate how the SZ signal corre- 
lates with other cluster properties, such as the mass, temperature and X-ray luminosity (the "scaling relations"), while departure from scaling relations indicate non-gravitational and non-thermal heating processes in strongly merging systems.

The Planch 1 space telescope designed for the study of CMBR and its anisotropy, has recently also been used for studying the SZ decrement (Planck Collaboration 2011a). The ongoing Planck SZ survey provides sky images at nine frequencies in the range $30-857 \mathrm{GHz}$ and is the first all-sky survey capable of blindly searching for distant and massive clusters, since the ROSAT all-Sky Survey (RASS) in the X-ray domain.

A sample of SZ detected clusters in the Planck Early Release Catalog (ESZ) is described in detail in Planck Collaboration (2011b). The highest signal-to-noise ratio (S/N > 6) sample of 189 clusters is derived from a blind multi-frequency search in the all-sky maps from the first 10 months of observations. Out of these, 20 are new, hitherto unknown clusters which are above the ESZ high signal-to-noise selection criterion. Twenty-five clusters have been observed to date with XMM-Newton in snapshot exposures $(\sim 10-20 \mathrm{ks})$, with 10 in the lower $\mathrm{S} / \mathrm{N}$ ratio category $(4<\mathrm{S} / \mathrm{N}<6)$ and 15 in high-significance range with $\mathrm{S} / \mathrm{N}$ $>5$. A total of 21 cluster candidates are validated as extended X-ray sources, the majority of which show highly irregular and dynamically perturbed structure of merging systems (Planck Collaboration 2011c).

In this Letter we report the discovery of giant $(>1 \mathrm{Mpc})$ non-thermal peripheral double radio relics of an unprecedented scale (separation $\sim 4.4 \mathrm{Mpc}$ at redshift 0.39 ) in a massive galaxy cluster PLCK G287.0+32.9, detected solely through a blind SZ search by Planck. In view of its extreme X-ray properties and non-relaxed morphology, we have imaged it with the Giant Metrewave Radio Telescope (GMRT), and also obtained deep optical images with the $2 \mathrm{~m}$ telescope at the IUCAA Girawali Observatory (IGO). These observations have revealed this cluster to be highly unusual in terms of its morphology, mass, richness and non-thermal emission, relating to its complex dynamical state.

We adopt a $\Lambda$ CDM cosmology with $H_{0}=71 \mathrm{~km} \mathrm{~s}^{-1} \mathrm{Mpc}^{-1}, \Omega_{M}=0.27$ and $\Omega_{\Lambda}=0.73$, resulting in scale of $5.26 \mathrm{kpc} \operatorname{arcsec}^{-1}$ (or 3.17 arcmin $\mathrm{Mpc}^{-1}$ ) for a redshift 0.39. Below $M_{n}$ and $R_{n}$ denote the total mass and radius corresponding to a total density contrast $n \rho(z)$, where $\rho(z)$ is the critical density of the universe at redshift $z$.

\footnotetext{
${ }^{1}$ Planck (http://www.esa.int/Planck) a project of the European Space Agency (ESA) with instruments provided by two scientific consortia funded by ESA member states (in particular the lead countries France and Italy), with contributions from NASA (USA) and telescope reflectors provided by a collaboration between ESA and a scientific consortium led and funded by Denmark.
} 


\section{PLCK G287.0+32.9 : An Exceptionally Massive and Hot Planck ESZ Cluster}

Our target PLCK G287.0+32.9 is the second most significant detection ( $\mathrm{S} / \mathrm{N}=10.2$ ) among the 20 new clusters of the Planck ESZ sample (Planck Collaboration 2011b). A $10 \mathrm{ks}$ $\mathrm{X}$-ray validation exposure taken with $X M M$-Newton reveals an extremely hot $\left(T_{x}=12.86\right.$ $\mathrm{keV})$, luminous $\left(L_{(0.1-2.4} \mathrm{keV}\right)=1.72 \times 10^{45} \mathrm{erg} \mathrm{s}^{-1}$ within $\left.R_{500}\right)$, and massive (estimated $M_{500}=1.57 \times 10^{15} M_{\odot}$ ) galaxy cluster at redshift of $z=0.39$ (from Fe K line fitting). PLCK G287.0+32.9 is also the most massive and the hottest new SZ cluster detected by Planck. Our observations with the $2 \mathrm{mt}$ telescope (see below) provide the first deep optical image, confirming it to be a very rich galaxy cluster. The X-ray and SZ properties are summarised in Table 1, taken from Planck Collaboration (2011c). We have reanalyzed the public domain XMM-Newton data providing clear evidence for significant morphological disturbances in the center, thus characterizing PLCK G287.0+32.9 as a dynamically perturbed, merging system (Figure 1).

\section{Observations}

\subsection{Optical Validation of Galaxy Cluster PLCK G287.0+32.9}

We observed PLCK G287.0+32.9 with the 2m optical telescope of the IUCAA Girawali Observatory (IGO) in India, using the IUCAA Faint Object Spectrograph 83 Camera (IFOSC). IFOSC employs an EEV $2 \mathrm{~K} \times 2 \mathrm{~K}$ CCD giving a field of view of about $10.5 \times 10.5$ arcminutes. Observations were taken on 2011 February 3rd, 6th, 7th and 10th, in fair seeing (average $\sim 1^{\prime \prime}$ ) and dark sky conditions. The analysis was done in a standard way using IRAF. The final, combined deep R-filter image has a total exposure time of $4 \mathrm{hr}(12 \times 1200 \mathrm{~s})$, which shows a dense concentration of galaxies near the cluster center. Lacking a good magnitude calibration of the CCD images, we used the SuperCOSMOS Sky Survey for photometric information (Hambly et al. 2001). We obtained a plot of galaxy counts by including galaxies with ESO R-magnitude $m_{R}<20.5$ and counting in cells of $100^{\prime \prime}$ radius. At the centre a peak galaxy over density factor of $\sim 10$ was found, relative to the mean field galaxy density far away. Thus, we independently confirm PLCK G287.0+32.9 to be a very rich and massive galaxy concentration (see Figure 1). 


\subsection{GMRT and VLA Radio Images}

A search for diffuse non-thermal radio emission in and around the new Planck ESZ clusters was carried out, using the TIFR-GMRT Sky Survey (TGSS) database at $150 \mathrm{MHz}$. A central radio-halo/relic source and a peripheral giant double-relic were thus identified towards PLCK G287.0+32.9. These are corroborated by the $1.4 \mathrm{GHz}$ NRAO VLA Sky Survey images (NVSS; Condon et al. 1998), as described below.

The $150 \mathrm{MHz}$ image presented here was derived from the data of the ongoing TGSS 2 (S.K. Sirothia et al., in preparation). We reanalyzed the TGSS snapshots with ROBUST = 5 weights (AIPS++ software) in order to enhance the sensitivity to extended emission. This map with a beam of $36.84^{\prime \prime} \times 28.58^{\prime \prime}$ FWHM in $47.6^{\circ}$ position angle, has an rms noise of 3-6 mJy beam ${ }^{-1}$ (see Fig. 2). The $1.4 \mathrm{GHz}$ map in Figure 3 is reproduced from the NVSS.

\section{Results}

Our key result is the discovery of a pair of giant $(l>1 \mathrm{Mpc})$, non-thermal peripheral radio relics of an unprecedented scale (projected separation $\sim 4.4 \mathrm{Mpc}$ at $\mathrm{z}=0.39$ ) in a merging galaxy cluster of extreme properties (Figure 2 top row). Such Mpc-scale double-relics are rare and only nine cases have been reported. Of these, the double radio relic associated with the well known merging cluster Abell $3667(\mathrm{z}=0.0556)$ and having an overall size of $3.9 \mathrm{Mpc}$ is the largest known till now (Röttgering et al. 1997). Furthermore, the present case (PLCK G287.0+32.9) is not only the first clear detection of diffuse non-thermal radio emission in a galaxy cluster detected solely through a blind SZ search by Planck, it is also the most distant $(z=0.39)$ giant double radio relic known, even surpassing the hitherto known case of the $z=0.27$ filamentary cluster $\mathrm{ZwCl} 2341.1+0000$ (van Weeren et al. 2009). In PLCK G287.0+32.9 the pair of Mpc-scale, sharp "arc" like relics are seen to the north and south of the cluster, capping the opposite ends of the X-ray emitting hot ICM and the central optical galaxy concentration (Figures 1 and 2 top row). The two extended radio-relics are marked $\mathrm{RN}$ (north relic) and $\mathrm{RS}$ (south relic), also shown with iso-contours, superposed on the optical images (lower left and right panels in Figure 2). Another Mpcscale Y-shaped filamentary radio feature is visible closer to the north of the cluster center, whose nature is presently unclear ( R.A. $11^{h} 50^{m} 51.30^{s}$, decl. $-28^{d} 02^{m} 57.4^{s}$ ), but which may well be another radio relic, as it has no obvious association with any galaxy. Both this feature and the peripheral double relic are visible also in the lower resolution NVSS image

\footnotetext{
${ }^{2}$ For TGSS survey details, online data and analysis please refer to http://tgss.ncra.tifr.res.in
} 
at $1.4 \mathrm{GHz}$ (Figure. 3), lending independent support to their being real. Moreover, NVSS image shows some diffuse radio emission near the cluster center, probably representing a cluster wide radio-halo (Figure 3). Unfortunately, the poor resolution and the presence of several discrete radio sources in that region makes it difficult to unambiguously visualise the proposed halo.

Note that the strong point source marked "S" on NVSS image (181 mJy peak) lacks a TGSS counterpart, implying a strongly inverted spectrum below $\sim 1 \mathrm{GHz}$. On the other hand, the nature of the extended Mpc-scale feature marked ' $F$ ' remains unclear; although seen in the TGSS $150 \mathrm{MHz}$ image (Figure 2), it is absent in the NVSS $1.4 \mathrm{GHz}$ map. This suggests that either it is a very steep spectrum radio-relic, possibly a southern counterpart to the inner relic, or merely a noise feature. To clarify this we have applied for much deeper GMRT imaging.

Integrated flux densities of the radio relics are; $550 \pm 50 \mathrm{mJy}(\mathrm{RN})$ and $780 \pm 50 \mathrm{mJy}$ (RS) at $150 \mathrm{MHz}$ (GMRT), and $33 \pm 5 \mathrm{mJy}(\mathrm{RN})$ and $25 \pm 5 \mathrm{mJy}(\mathrm{RS})$ at $1400 \mathrm{MHz}$ (Very Large Array, VLA). Thus, the radio spectral index $\alpha$ between the two frequencies is extremely steep, i.e., 1.26 for the relic RN and 1.54 for the relic RS. The corresponding value for the giant Y-shaped relic candidate north of the cluster center is also very steep at $\alpha=1.20$. These very steep spectra support the interpretation of all these giant diffuse sources as being radio relics, an inference corroborated by the lack of obvious association with any prominent galaxy (Figure 2). We also point out that the radio emission from a foreground $\left(m_{R}=15.34\right)$, emission-line galaxy 2MASX J11504002-2800582 at $z=0.0605$ (Mauch and Sadler 2007) is seen superposed on the western end of the relic RN.

Our reanalysis of the $10 \mathrm{ks}$ exposure XMM-Newton archival data for PLCK G287.0+32.9 shows an extremely X-ray luminous cluster having prominent substructure within the central $1.5^{\prime}$ ( $\sim 500 \mathrm{kpc}$ ) core region (Figure 1). The brightest central elliptical galaxy (ESO R magnitude 17.56$)$ at R.A. $11^{h} 50^{m} 50.1^{s}$, Dec. $-28^{d} 04^{m} 55.3^{s}$ is located within a subcluster, which is clearly shifted to the south-east from the main X-ray peak by $\sim 1.3^{\prime}$ (400 kpc). The compression of the X-ray contours north-west of the main X-ray peak indicates ongoing gas motions, either due to gas "sloshing" following a merger, or motion of a merging subcluster unit. A detailed analysis of the X-ray data will be presented in J. Bagchi et al. (in preparation). 


\section{Discussion and Conclusions}

The giant radio relics reported here in the cluster PLCK G287.0+32.9 are not only the first such detections for an SZ cluster survey, but also this relic pair has the largest separation (4.4 Mpc) and redshift $(z=0.39)$ compared to any other giant double relic known. In PLCK G287.0+32.9 the projected distances from the X-ray center of the cluster are about $5^{\prime}$ (or $1.58 \mathrm{Mpc}$ ) for the northern relic and 9.5' (or $3 \mathrm{Mpc}$ ) for the southern relic. The virial radius $\left(R_{v i r}\right.$ ) of cluster can be estimated by taking $R_{v i r} \approx R_{200}$ and scaling from $R_{500}$, i.e., $R_{200}$ $=R_{500} \times(500 / 200)^{1 / 3} \times\left(M_{200} / M_{500}\right)^{1 / 3}$. This gives $R_{200} \approx 2.3 \mathrm{Mpc}$, using $R_{500}=1541 \mathrm{kpc}$ and the mean value of mass ratio $\left(M_{200} / M_{500}\right)=1.40 \pm 0.02$, obtained from the Navarro-FrenkWhite (NFW) profile fit to nearby $(z \lesssim 0.15)$ clusters (Pointecouteau et al. 2005). Thus the separation from the cluster centre of the relics is about $1.3 \times R_{200}$ for $\mathrm{RS}$ and about $0.7 \times R_{200}$ for RN. The mechanism of electron acceleration at such extreme distances from the cluster center is little understood. According to a currently popular interpretation, double-relics are the sites where cosmic-ray particles are accelerated in-situ to relativistic energies by diffusive first-order Fermi acceleration, such that the resulting radio emission traces the outward expanding powerful shock fronts originating from a major cluster merger activity at the center (Enßlin et al. 1998; Bagchi et al. 2006; Hoeft et al. 2008; van Weeren et al. 2010).

This scenario is strongly supported by the extremely high temperature and unrelaxed X-ray appearance of PLCK G287.0+32.9. A recent hydro-simulation of major mergers (Paul et al. 2011) show that the emergent ellipsoidal shock front may expand well beyond the virial radius and the brightest parts of it are roughly tangential to the principal merger axis, thus resembling a pair of concave "arcs", akin to those found in PLCK G287.0+32.9 . As the shock front crosses the virial radius, it begins to interact with the intergalactic matter filaments, resulting in its fragmentation into segments. Such predicted evolution of the peripheral shocks appears to be mirrored in both members of the relic pair, whose overall arc-shaped radio morphology exhibits one or two distinct emission gaps (Figure 2). Thus, such giant radio relics can be a new probe of the large-scale cosmic structures (see also Bagchi et al. 2006; van Weeren et al. 2010; Brown et al. 2011).

XMM-Newton data confirm that the cluster PLCK G287.0+32.9 is exceptional in terms of its mass, temperature, and luminosity (Table 1), detected with high S/N by Planck (Planck Collaboration 2011d). Given the perturbed dynamical state of merging system PLCK G287.0+32.9, it is of interest to investigate its position on the well-known X-raySZ scaling planes. As shown in (Planck Collaboration 2011c), indeed PLCK G287.0+32.9 is a prominent outlier from both $L_{x, 500}-Y_{500}$ and $M_{500}-L_{x, 500}$ mean scaling lines, which are obtained from the bias corrected local REXCESS X-ray sample (Arnaud et al. 2010), and a sample of 62 nearby galaxy clusters detected by Planck showing strong SZ decrement 
(Planck Collaboration 2011d). Although due to the intrinsic scatter in these correlations, the deviation of PLCK G287.0+32.9 from these scaling laws is not more than $1 \sigma$, nevertheless it is noticeably underluminous in X-rays for its large mass, in common with other new extreme low luminosity, high mass, disturbed clusters that are being revealed by Planck.

Synchrotron emission from the giant relic pair shows that magnetic fields of appreciable strength are present not only in the ICM but also in the diffuse intergalactic medium beyond, i.e., in the gas that will be shocked as it accretes onto collapsing structures - the precursors of virialized galaxy clusters. Such magnetic fields are also required for providing the scattering centers for the diffusive shock acceleration mechanism. As it is not obvious how magnetic fields are amplified up to the requisite large values along the filaments, studies similar to the one reported here pose a new challenge to theoretical models. The detection of large scale diffusive shocks via synchrotron radio emission in the sparsely studied, rarefied intergalactic field around the cluster-outskirts, i.e., beyond the virial radius $\left(R \gtrsim R_{200}\right)$, is a very significant result, and it provides a foretaste of science with the upcoming low frequency radio telescopes like LOFAR and LWA (and SKA at higher frequency), as an effective probe of the non-thermal processes in merging clusters and within the filamentary cosmic-web of the near and distant parts of the universe (e.g. Rudnick et al. 2009).

We thank the staff of the GMRT and IUCAA Girawali Observatory that made these observations possible. We thank Monique Arnaud and Gabriel Pratt for useful discussions. GMRT is run by the National Centre for Radio Astrophysics (NCRA) of the Tata Institute of Fundamental Research (TIFR). The X-ray data are from XMM-Newton, a European Space Agency (ESA) science mission with instruments and contributions directly funded by ESA Member States and NASA. MBP was supported by the "INSPIRE Fellowship" program of DST, Ministry of Science and Technology, Government of India. N.W. was supported by the NASA through Chandra Postdoctoral Fellowship Award Number PF8-90056 issued by the Chandra X-ray Observatory Center, which is operated by the Smithsonian Astrophysical

Observatory for and on behalf of the National Aeronautics and Space Administration under contract NAS8-03060.

\section{REFERENCES}

Arnaud, M., et al. 2010, A\&A, 517, A92

Bagchi, J., et al. 2006, Science, 314, 791

Brown, S., Duesterhoeft, J., \& Rudnick, L. 2011, ApJ, 727, L25 
Brunetti, G., \& Lazarian, A. 2007, MNRAS, 378, 245

Condon, J.J., et al. 1998, AJ, 115, 1693

Dennison, B. 1980, ApJ, 239, L93

Enßlin, T.A., Biermann, P.L., Klein, U., Kohle, S. 1998, A\&A, 332, 395

Ferrari, C., et al. 2008, Space Sci. Rev., 134, 93

Govoni, F., Markevitch, M., Vikhlinin, A., van Speybroeck, L., Feretti, L., \& Giovannini, G. 2004, ApJ, 605, 695

Hambly, N.C., et al. 2001, MNRAS, 326, 1279

Hoeft, M., et al. 2008, MNRAS, 391, 1511

Mauch, M., \& Sadler, E.M. 2007, MNRAS, 375, 931

Paul, S., Iapichino, L., Miniati, F., Bagchi, J., \& Mannheim K. 2011, ApJ, 726, 17

Planck Collaboration 2011a, Planck early results 01: The Planck Mission, A\&A in press, (arXiv:1101.2022)

Planck Collaboration 2011b, Planck early results 08: The All-sky Early Sunyaev-Zeldovich Cluster Sample, A\&A in press, (arXiv:1101.2024)

Planck Collaboration 2011c, Planck early results 09: XMM-Newton Follow-up for Validation of Planck Cluster Candidates, A\&A in press, (arXiv:1101.2025)

Planck Collaboration 2011d, Planck early results 11: Calibration of the local galaxy cluster Sunyaev-Zel'dovich scaling relations, A\&A in press, (arXiv:1101.2026)

Pointecouteau, E., Arnaud, M., \& Pratt, G.W 2005, A\&A, 435, 1

Röttgering, H., Wieringa, M. H., Hunstead, R. W., \& Ekers, R. D. 1997, MNRAS, 290, 577

Rudnick L., et al., 2009, Astro2010: The Astronomy and Astrophysics Decadal Survey, Science White Papers, No. 253

Sunyaev, R.A. \& Zel'dovich, Y.B. 1972, Comments Astrophys. Space Phys., 4, 173

Sunyaev, R.A., \& Zel'dovich, Y.B. 1980, ARA\&A, 18, 573

Vanderlinde, K., et al. 2010, ApJ, 722, 1180 
van Weeren, R.J., et al., 2009, A\&A, 506, 1083

van Weeren, R.J., et al., 2010, Science, 340, 347

Williamson, R., et al. 2011, ApJ submitted, (arXiv:1101.1290) 
Table 1:

X-ray and SZ Parameters ${ }^{a}$

Right Ascension

$11^{h} 50^{m} 49.20^{s}$

Declination

$-28^{d} 04^{m} 36.5^{s}$

Redshift ${ }_{-}^{b}$

0.39

$\mathrm{X}$-ray temperature

$12.86 \pm 0.42 \mathrm{keV}$

X-ray luminosity_c

$17.20( \pm 0.11) \times 10^{44} \mathrm{ergs}^{-1}$

Cluster mass

$R_{500}$ $15.72( \pm 0.27) \times 10^{14} M_{\odot}$

SZ Compton $Y_{500}{ }^{d}$ $1541 \mathrm{kpc}$

X-ray $Y_{X, 500} \stackrel{e}{=}$

${ }^{a}$ Planck Collaboration (2011b, 2011c)

${ }^{b}$ From fitting Fe-K lines

${ }^{c}$ Within $R_{500}$, in $(0.1-2.4 \mathrm{keV})$ band

${ }^{d}$ Spherically integrated Compton $Y$ parameter measured interior to $R_{500}$

${ }^{e} \mathrm{X}$-ray derived $Y$-parameter. $\quad Y_{X, 500}=$ $M_{g, 500} T_{x}$, the product of gas mass within $R_{500}$ and X-ray temperature 

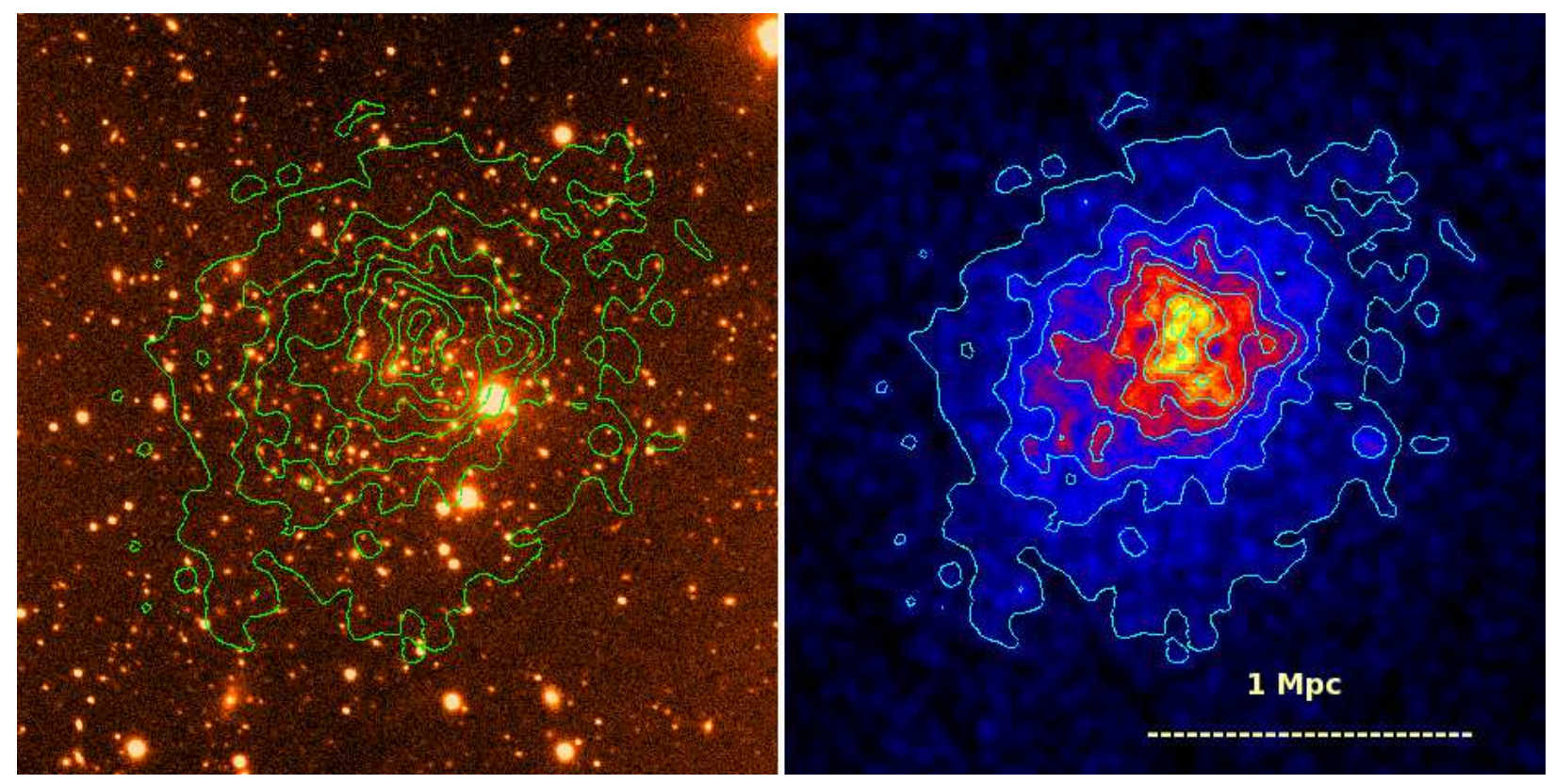

Fig. 1.- Left: deep $R$-band image taken with the $2 \mathrm{~m}$ telescope of IUCAA. The FOV is 7 arcmin on each side $(\sim 2.2 \mathrm{Mpc})$. X-ray contours are from XMM-Newton $(0.3-10 \mathrm{keV}$, MOS1/2, and PN detectors, binned and smoothed). Right: the same X-ray image is shown along with iso-contours. 


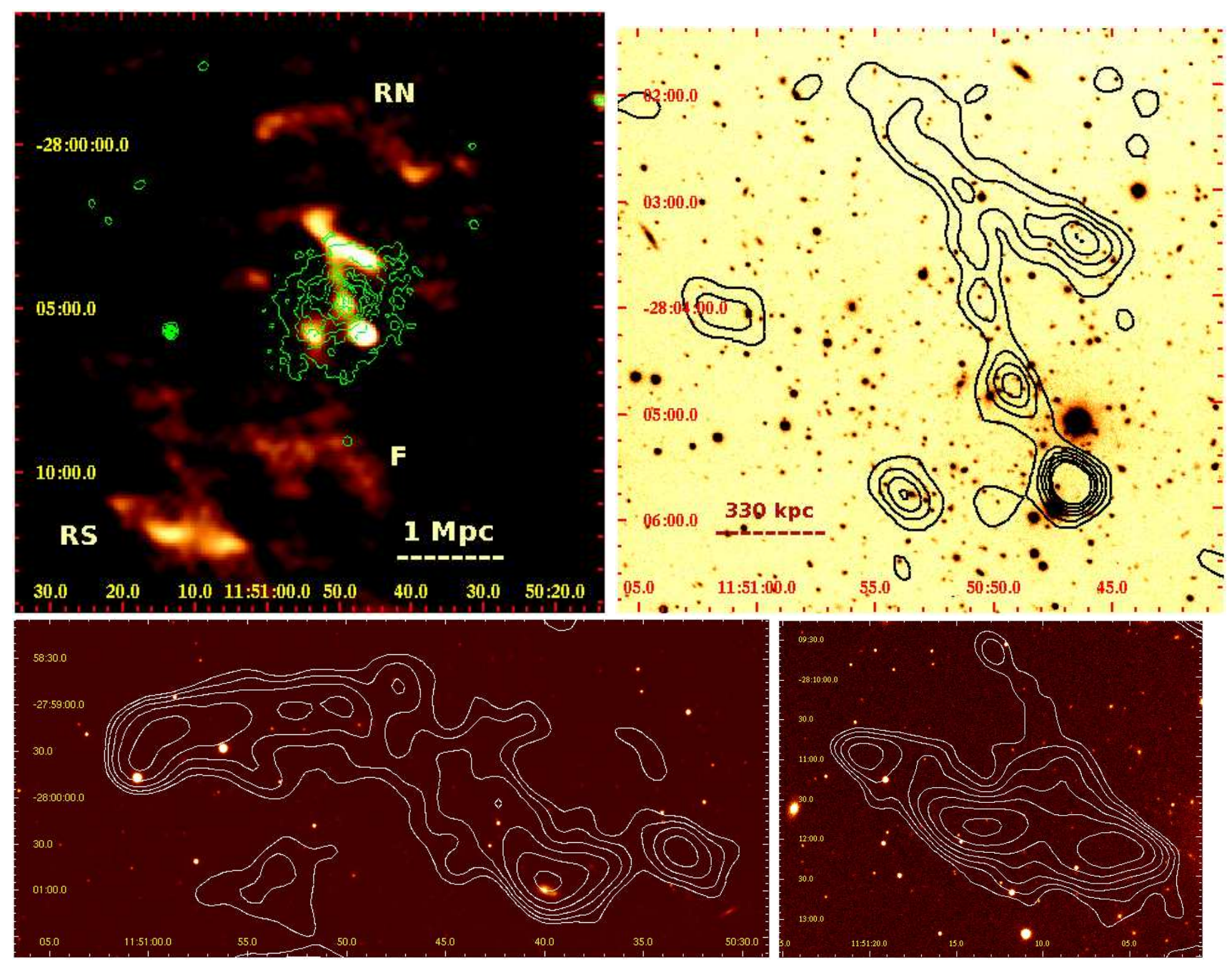

Fig. 2.- Upper left: GMRT $150 \mathrm{MHz}$ radio map of the cluster (orange-yellow, beam FWHM $36.84^{\prime \prime} \times 28.58^{\prime \prime}$ in $47.6^{\circ}$ P.A.). Superposed are the XMM-Newton X-ray emission contours (green) in 0.3 - $10 \mathrm{keV}$ energy range. A pair of giant radio relics at the cluster periphery are marked RN (north relic) and RS (south relic). Upper right: GMRT $150 \mathrm{MHz}$ radio map near the cluster center (beam FWHM $24^{\prime \prime} \times 15^{\prime \prime}$ in P.A. 30 ), superposed on the optical $R$-band image. Contour levels are: 7.5, 13.57, 19.64, 25.7, 31.75, 43.92, and $50 \mathrm{mJy} \mathrm{beam}^{-1}$. Lower left and right: Contour plots of radio relics RN (left) and RS (right) at $150 \mathrm{MHz}$, superposed on the R-band image of the region. The beam FWHM is $36.84^{\prime \prime} \mathrm{x} 28.58^{\prime \prime}$ in $47.6^{\circ}$ P.A.. Contour levels are: 10, 13, 17, 22, 29, 33, and $50 \mathrm{mJy}^{\text {beam }}{ }^{-1}$ with typical rms noise $\sim 3-5$ mJy beam $^{-1}$. 


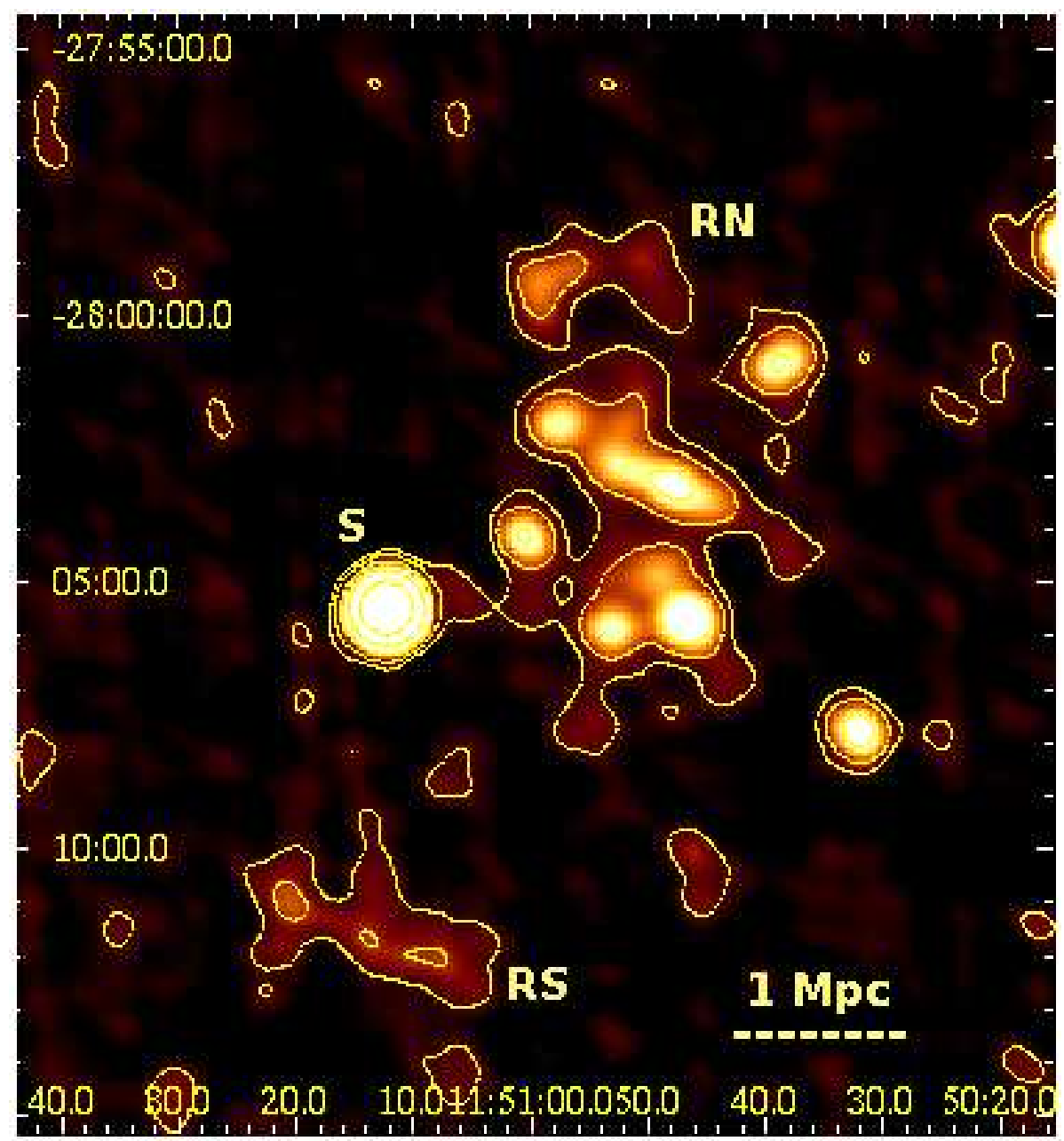

Fig. 3.- Radio image from VLA $1.4 \mathrm{GHz}$ NVSS at $45^{\prime \prime}$ FWHM resolution. Contours are logarithmically spaced between 0.9 to $60.0 \mathrm{mJy}^{\text {beam }}{ }^{-1}$ and the noise rms is $\sim 0.45 \mathrm{mJy}$ beam $^{-1}$. The pair of peripheral radio relics are marked RN and RS. Compact source "S" is discussed in the main text. 